Research Article

\title{
Enhanced Long-term Antithrombogenicity Instigated by Covalently- Attached Surface Modifier on Biomedical Polymers
}

Kiril Fedorov ${ }^{1,2}$, Sonia Sheikh ${ }^{1}$, Alex Romaschin ${ }^{3}$, Michael Thompson ${ }^{1,}{ }^{*}$

1. Department of Chemistry, University of Toronto, 80 St. George Street, Toronto, Ontario M5S 3H6, Canada; E-Mails: kiril.m.fedorov@gmail.com; ssheikh.chem@gmail.com; m.thompson@utoronto.ca

2. Institute of Biomaterials and Biomedical Engineering, University of Toronto, 164 College Street, Toronto, Ontario, M5S 3G9, Canada

3. Keenan Research Centre and Clinical Biochemistry, St. Michael's Hospital, 30 Bond Street, Toronto, Ontario, M5B 1W8, Canada; E-Mail: alexander.romaschin@unityhealth.to

* Correspondence: Michael Thompson; E-Mail: m.thompson@utoronto.ca

Academic Editor: Hossein Hosseinkhani

Special Issue: Applications and Development of Biomaterials in Medicine

Recent Progress in Materials

2020, volume 2, issue 3

doi:10.21926/rpm.2003019
Received: May 03, 2020

Accepted: August 12, 2020

Published: August 18, 2020

\begin{abstract}
During a variety of medical procedures such as renal dialysis, bypass surgery, and lung transplantation patient blood is exposed to the surface of a number of polymeric materials such as polycarbonate (PC), poly (vinyl chloride) (PVC) and polysulfone (PS) for a period up to several days. Such exposure may result in undesirable protein-material interactions that can potentially trigger deleterious biological processes including thrombosis, which may be responsible for other complications such as cognitive disability. In order to address this issue, we have further examined the behavior of an ultrathin antifouling and antithrombogenic coating based on monoethylene glycol silane surface chemistry. Samples of polymeric substrates were exposed to
\end{abstract}

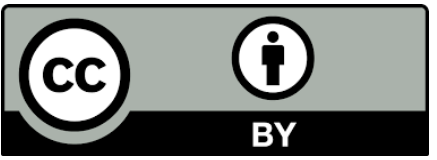

(C) 2020 by the author. This is an open access article distributed under the conditions of the Creative Commons by Attribution License, which permits unrestricted use, distribution, and reproduction in any medium or format, provided the original work is correctly cited. 
blood flow at a shear rate of $\sim 20 \mathrm{~s}^{-1}$ for time periods of 3 and 6 hours, as well as 3 days. No additional anticoagulant chemistry was applied in the experiments. For all time periods, platelet adhesion, aggregation, and thrombus formation on the coated surfaces was inhibited compared to bare substrates (coated PC performing the best, followed by PVC-, and then PS-coated), strongly supporting the results of previous research conducted over far shorter blood contact times.

\section{Keywords}

Blood; antithrombogenicity; hemocompatibility; organosilane adlayer; medical polymers; polycarbonate; poly (vinyl chloride); polysulfone

\section{Introduction}

Annually, approximately 3000 surgeries are performed per 100,000 head of population, which translates to around $\sim 232$ million operations worldwide [1]. As the world population continues to grow in tandem with the expected overall rise in life expectancy and age, the number of surgeries is anticipated to increase significantly, at least according to the current trend [1]. Many such surgeries and other medical procedures require associated medical equipment, instruments and circuitry to come in contact with bodily fluids including blood, which may stimulate biological processes notably those orchestrated by the immune and coagulation systems resulting in highly deleterious outcomes [2-5]. Indeed, postoperative complications such as organ dysfunction are an unfortunate reality. A particularly serious example is the indication that blood-surface interaction during hemodialysis and bypass surgery is linked to incapacitating brain disorder or cognitive disability [6, 7]. The implication here is that clots - formed at the hemo-incompatible surface of biomedical equipment - are transferred to the brain, where they lodge within blood vessels obstructing vital blood supply in the process. This cerebrovascular accident (or "stroke"), in turn, affects brain competence. One crucial consideration with respect to biological fluid-foreign material interaction is the duration of the specific procedure or surgery to be performed. This parameter will have a significant effect since higher contact times are very likely to result in an increased probability of medical complications. Typical durations for various procedures are as follows - apheresis (60-120 minutes) [8], hemodialysis (1-5 hours) [9, 10], hemofiltration (8-12 hours) [11], cardiopulmonary bypass surgery and extracorporeal membrane oxygenation (1-6 hours) [12]. In summary, most surgeries and other medical procedures commonly require 3 or fewer hours, but it is not rare that 6 or more hours may be needed.

In addition to the extracorporeal configurations mentioned above, there also exist issues associated with the ubiquitous in vivo employment of catheters (aside from the well-known and -characterized occurrence of infection introduced by such medical equipment). For example, the indwelling central venous catheter (CVC) has extensively been linked to thrombosis and has been reported in up to $33 \%$ of catheter uses, as well as up to 21 episodes/1000 catheter-days [13]. Other less common complications that may be related to the treatment of occluded catheters include intracranial 
hemorrhage (ICH), major bleeding (MB), and embolism [14-16]. Other cases may result in systemic inflammation and accelerated arteriosclerosis. In view of the various deleterious medical consequences connected to biological fluid-surface interactions occurring daily in a great number of routine medical procedures/surgeries, there has been a long-standing need for the introduction of substrate interfacial modification that is capable of the provision of enhanced biocompatibility.

In the present paper, the hemocompatibility focus is on synthetic polymeric materials that are employed extensively both in vivo and extracorporeally. Due to their various intrinsic properties, such as flexibility and chemical stability, different polymers find variable applications in medical procedures [17]. Examples are poly (vinyl chloride) (PVC), which has been employed very widely for the fabrication of blood conduits, blood bags, catheters, and endotracheal tubes, as well as polycarbonate (PC), which is used as a container material for equipment associated with bypass surgery [18, 19]. In order to enhance the biocompatibility of polymeric (and other) materials, an enormous number of surface modifications and treatments have been attempted over a number of years; these are comprehensively reviewed in a recent book by Thompson et al. [20]. In addition to physical modification such as plasma treatment, a plethora of coatings have been studied including "bio-inspired" constructs based on single amino acids or longer peptides and peptoids, oligo- and polyethylene oxides/glycols, zwitterionic sulfoand carboxybetaines and complex hybrids or derivatives of these chemical systems [21-23].

Recently, in our own work, we have successfully derivatized the surfaces of both PC and PVC with an ultra-thin (nanometer-thick), monoethylene glycol-based (MEG-OH) nanogel siloxane adlayer [24]. Regarding PC, the antithrombogenicity of this material was assessed in real-time using a perfusion chamber and fluorescently-labelled whole human blood flown for 5 minutes at a controlled shear rate of $1000 \mathrm{~s}^{-1}$. Remarkably, platelet adhesion, aggregation, and thrombus formation on the MEG-OH coating were greatly inhibited ( $>97 \%$ decrease in surface coverage) compared to the bare substrate [25]. In a similar vein, a decrease in surface coverage by platelet aggregation in the PVC study exceeded $99 \%$, for all the investigated shear rates $\left(300,900\right.$, and $\left.1500 \mathrm{~s}^{-1}\right)$ [26]. Physico-chemical studies of the MEG-OH adlayer on $\mathrm{Si} / \mathrm{SiO}_{2}$ by neutron reflectometry [27] and molecular dynamic simulations [28] have suggested that the antifouling behavior of the adlayer is connected to surface hydration and how water organizes within and atop the film. The intrinsic water domain displays restricted molecular motion, which extends into bulk water to form a physically-distinct interfacial phase. The term "kosmotropic effect" has been used in this context.

In the present paper, we expand the scope of our antithrombogenic investigation and describe experiments wherein blood is exposed to MEG-OH-coated PC, PVC and polysulfone (PS) (Figure 1) over relatively long periods of time (up to 3 days). With regard to platelet integrity, it is known that platelets can indeed be stored at $22.0^{\circ} \mathrm{C}$ displaying a half-life of around 4 days. Thus, even in the longest experiment we conducted, it is very likely that the majority of the platelets are still alive after 3 days. This work demonstrates that blood incompatibility is not an issue for MEG-OH-coated PC, PVC, and PS when exposed to blood for lengthy time periods. 


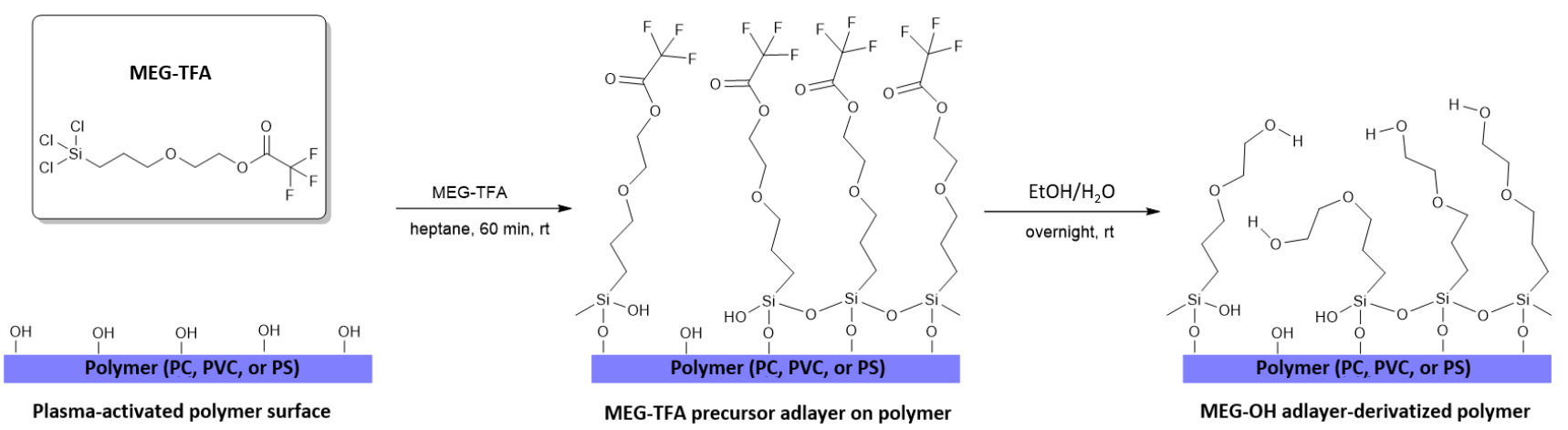

Figure 1 Schematic representation of the straightforward, two-step surface modification of polymer (PC, PVC, or PS) surface with antithrombogenic MEG-OH surface chemistry.

\section{Materials and Methods}

\subsection{Materials}

PC, PVC, PS (sheet) were purchased from McMaster-Carr (Elmhurst, USA). Sodium dodecyl sulfate (SDS), ethanol (95\%), Dulbecco's phosphate buffered saline (PBS, $\mathrm{CaCl} 2$ and $\mathrm{MgCl} 2$ free $-\mathrm{pH} 7.4$ ), and 3, 3'-dihexyloxacarbocyanine iodide $\left(\mathrm{DiOC}_{6}\right)$, were all purchased from Sigma-Aldrich, Canada and used as received. Heptane was obtained from Caledon Laboratories, Georgetown, Canada. The MEG-TFA precursor molecule for MEG-OH surface modification was synthesized as described previously [29].

\subsection{Polymer Substrate Cleaning and Activation}

PC, PVC, and PS substrates $(0.5 \times 0.9 \times 0.16 \mathrm{~cm}$ in size $)$ were rinsed under tap water and sonicated for 1 minute to remove any major residue. The polymer substrates were then washed in $1 \%$ SDS (prepared in tap water), sonicated for 5 minutes in 1\% SDS, and then successively rinsed in $1 \%$ SDS $(\times 1)$, tap water ( $\times 3$ ), and finally $95 \%$ ethanol (copiously). The polymer substrates were then dried under $\mathrm{N}_{2}$ gas and placed into a plasma cleaner (13.56 MHz, 30W, $150 \mathrm{mTorr}$ ) for various durations (depending on the polymer under study). After plasma cleaning, the polymer substrates were placed into a humidity chamber ( $80 \%$ relative humidity, room temperature) for overnight surface hydration.

\subsection{Polymer Surface Modification with MEG-OH Adlayer}

MEG-TFA surface modifier was diluted with heptane in a glove box kept under inert $\left(\mathrm{N}_{2}\right)$ and anhydrous $\left(\mathrm{P}_{2} \mathrm{O}_{5}\right)$ atmosphere. Cleaned/activated polymer substrates were then individually placed in pre-silanized glassware that was subsequently filled with a $1 \mu \mathrm{L} / \mathrm{mL}$ solution of MEG-TFA/heptane (at a ratio of $1 \mathrm{~mL} / 1 \mathrm{~cm}^{2}$ solution volume/substrate surface area) before being placed on a rotary shaker at low speed for 1 hour. After treatment, the samples were removed from the glove box and then successively rinsed with heptane $(\times 3)$, sonicated for 5 minutes in heptane, and then rinsed once more with this solvent. This rinsing procedure was repeated with $95 \%$ ethanol, before drying the samples under a gentle stream of $\mathrm{N}_{2}$ gas. To convert MEG-TFA adlayers into MEG-OH surfaces, the samples were 
individually soaked in $1 \mathrm{~mL}$ of a $1 / 1(\mathrm{v} / \mathrm{v})$ solution of $95 \%$ ethanol and tap water overnight at room temperature. Finally, the MEG-OH-coated surfaces were rinsed in $95 \%$ ethanol $(\times 3)$, dried under a gentle $\mathrm{N}_{2}$ stream, and then stored in sealed containers awaiting analysis/blood contact experiments.

\subsection{X-ray Photoelectron Spectroscopy and Contact Angle Goniometry Adlayer Characterization}

X-ray photoelectron spectroscopy (XPS) was performed with a Theta Probe Instrument (ThermoFisher Scientific). Samples were analyzed with monochromated Al Ka X-rays at a take-off angle of $90^{\circ}$ relative to the surface. Avantage software (ThermoFisher Scientific) was used to perform peak fitting and data analysis with binding energy being calibrated to the main $C_{1 s}$ signal at $285 \mathrm{eV}$. Static contact angles were measured with a CAM101 goniometer (KSV Instruments) and deionized water $(18.2 \mathrm{M} \Omega \mathrm{cm})$ as the test liquid.

\subsection{Blood Contact Experiments}

Whole blood was collected in Vacutainers from volunteers with no known blood disorders and labelled with $\mathrm{DIOC}_{6}$ as described previously $[25,26]$. The blood from the same volunteer was used for the experiments for equal amounts of control and MEG-OH-coated surfaces to avoid bias. A tubular flow chamber was built in house to expose surfaces to fluorescently-labelled blood and employed in a dark room to avoid any possible photobleaching. PC, PVC, or PS substrates - bare or MEG-OH-coatedwere individually inserted into the flow-through chamber and whole blood flown over at a shear rate of $\sim 20 \mathrm{~s}^{-1}$ (in a closed-loop system) using a peristaltic pump for 3 hours, 6 hours, as well as 3 days. All chambers were employed separately for the specific time periods, with each experiment being conducted with equivalent bare and MEG-OH samples. Substrates were then removed and gently rinsed in $1 \mathrm{~mL}$ PBS for 5 minutes. Next, the substrates were placed in $3 \%$ formaldehyde solution (in PBS) for 5 minutes and then removed and rinsed with PBS $(\times 3)$. Finally, the surfaces were stored in PBS and placed in a cold dark environment prior to microscope measurements.

\subsection{Fluorescence Microscopy}

Platelet adhesion, aggregation and thrombus formation was visualized with a BX61W1 upright microscope (Olympus, USA), equipped with a Rolera EM-C2 CCD camera (QImaging, Canada) with $8 \times 8$ $\mu \mathrm{m}$ pixel size. The required exposure time was set to $100 \mathrm{~ms}$ without camera gain. The excitation source used was an arc mercury lamp, X-Cite 120 PC fluorescence illumination system (EXFO Photonic Solutions Inc., USA). To filter fluorescent signals, a green 33 fluorescent filter cube set, FITC 3540B (Semrock, USA), which combines a $35 \mathrm{~nm}$ wide excitation bandpass filter centered at $482 \mathrm{~nm}$ and a 40 $\mathrm{nm}$ emission bandpass filter centered at $536 \mathrm{~nm}$, was used. $4 \times$ objective lens with a numerical aperture of 0.28 (Olympus, USA) were used with pixel sizes of $2 \mu \mathrm{m}$. Videos were collected using both MicroManager and ImageJ software. Platelet aggregate sizes were measured using ImageJ software in 2D mode. 


\section{Results and Discussion}

\subsection{Polymer Surface Characterization}

Prior to studying the interaction of polymer samples with blood, derivatized surfaces were assessed for successful MEG-OH modification using X-ray photoelectron spectroscopy (XPS) (Table 1) [18, 19]. Briefly, it is important to establish that XPS analysis of bare, unmodified polymer substrates displayed intense signals for carbon ( $\mathrm{C}_{1 \mathrm{~s}}$ at $\sim 285 \mathrm{eV}$ ), an element characteristic to PC, PVC, and PS substrates (Table 1). Following plasma activation, the relative intensity of this peak decreased, while that for oxygen $\left(\mathrm{O}_{1 \mathrm{~s}}\right.$ at $\left.\sim 532 \mathrm{eV}\right)$ increased since the polymer surfaces become activated with -OH groups (Table 1) [30-32]. As expected, silanization of PC, PVC, and PS substrates with oxygen-, silicon-, and fluorinebearing MEG-TFA surface modifier generated two new XPS peaks - one for silicon ( $\mathrm{Si}_{2 \mathrm{p}}$ at $\sim 103 \mathrm{eV}$ ) and another for fluorine $\left(F_{1 s}\right.$ at $\left.\sim 688 \mathrm{eV}\right)$ - as well as an increase in the relative intensity of the oxygen signal (Table 1). With respect to carbon, the XPS signal decreased substantially upon formation of the MEGTFA adlayer for all the polymers - as anticipated for the now buried substrate from which most of the carbon signal originates (Table 1). Upon conversion of MEG-TFA to MEG-OH adlayer, the fluorine XPS signal disappeared indicating that the labile terminal trifluoroacetyl (TFA) moieties had been completely cleaved upon solvolysis, while the signal for silicon was not affected demonstrating that the solvolytic treatment did not etch the residual MEG-OH coating from the PC, PVC, or PS substrates (Table 1). With respect to PS, the $S_{2 p}$ XPS signal ( $168 \mathrm{eV}$ ) was reduced for MEG-TFA/MEG-OH-coated surfaces (compared to bare PS), which is another indication of adlayer deposition, since the sulfur element is only attributed to PS (Table 1, Figure 2). Surface characterization with XPS concluded that all polymers employed in this study were successfully modified with MEG-OH adlayer.

Table 1 XPS relative atomic percentages for the characteristic elements of PC, PVC, and PS substrates (before and after plasma activation) and MEG-TFA/MEG-OH adlayers, and static contact angle measurements for these surfaces recorded with deionized water (triplicate analysis).

\begin{tabular}{|c|c|c|c|c|c|c|c|c|}
\hline & & \multicolumn{6}{|c|}{ Relative atomic percentage (\%) } & \multirow{2}{*}{$\begin{array}{l}\text { Contact } \\
\text { angle }\left({ }^{\circ}\right)\end{array}$} \\
\hline & & $\mathrm{C}_{1 \mathrm{~s}}$ & $\mathrm{Cl}_{2 \mathrm{p}}$ & $\mathbf{O}_{1 \mathrm{~s}}$ & $F_{1 s}$ & $\mathbf{S i}_{2 p}$ & $S_{2 p}$ & \\
\hline \multirow{4}{*}{ PC surface } & Bare & 82.8 & - & 15.4 & 0.4 & 0.5 & - & $94 \pm 2$ \\
\hline & Plasma-activated & 76.3 & - & 22.8 & 0.0 & 0.9 & - & $45 \pm 1$ \\
\hline & MEG-TFA adlayer & 44.7 & - & 36.8 & 5.9 & 12.6 & - & $85 \pm 1$ \\
\hline & MEG-OH adlayer & 47.5 & - & 39.0 & 0.0 & 13.5 & - & $55 \pm 2$ \\
\hline \multirow{4}{*}{ PVC surface } & Bare & 69.2 & 28.1 & 2.6 & 0.1 & 0.0 & - & $101 \pm 4$ \\
\hline & Plasma-activated & 62.8 & 27.5 & 9.7 & 0.0 & 0.0 & - & $62 \pm 2$ \\
\hline & MEG-TFA adlayer & 37.3 & 1.7 & 32.2 & 16.5 & 12.3 & - & $91 \pm 1$ \\
\hline & MEG-OH adlayer & 42.5 & 7.4 & 35.5 & 0.4 & 14.5 & - & $44 \pm 1$ \\
\hline \multirow{2}{*}{ PS surface } & Bare & 79.8 & - & 13.9 & 0.5 & 1.0 & 3.8 & $91 \pm 2$ \\
\hline & Plasma-activated & 70.9 & - & 23.0 & 0.3 & 0.8 & 4.0 & $22 \pm 2$ \\
\hline
\end{tabular}




\begin{tabular}{llllllll} 
MEG-TFA adlayer & 46.0 & - & 26.9 & 16.5 & 9.8 & 0.4 & $88 \pm 3$ \\
MEG-OH adlayer & 49.2 & - & 34.2 & 2.7 & 13.4 & 0.3 & $55 \pm 1$ \\
\hline
\end{tabular}

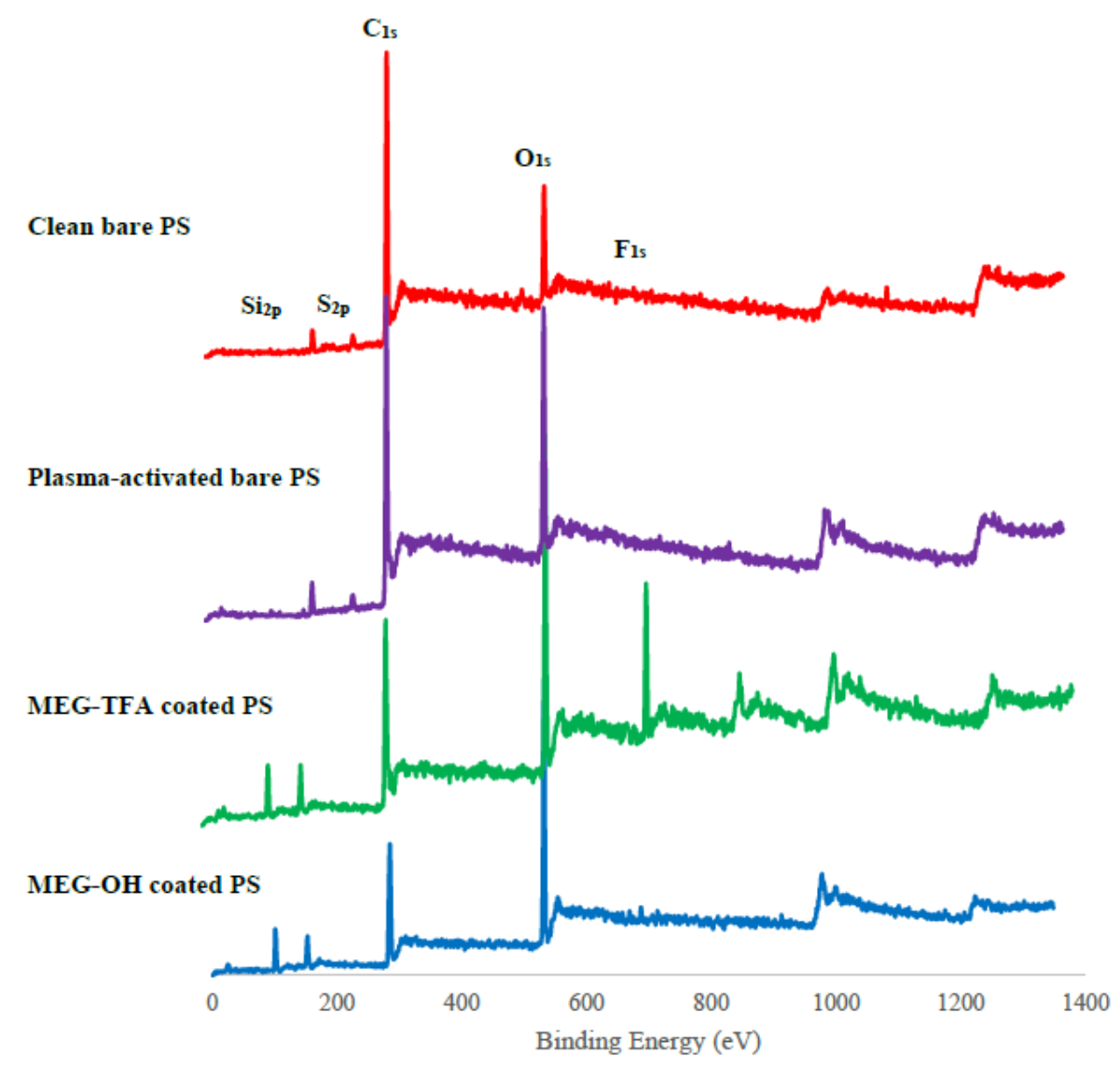

Figure 2 XPS surveys for (top to bottom) clean bare PS (red), plasma-activated bare PS (purple), as well as MEG-TFA (green) and MEG-OH (blue) coated PS. XPS surveys for bare and MEG-OH-coated PC and PVC are found in references 25 and 26, respectively.

To supplement XPS analysis, static contact angle (CA) measurements were also performed with water as the test liquid (Table 1). As received (i.e. non-cleaned and non-activated), bare substrates presented a rather hydrophobic surface with CA values ranging from 91 to $101^{\circ}$, depending on the polymer. Upon plasma cleaning and activation, the CA significantly decreased for all polymers, which is consistent with the formation of a more hydrophilic surface presenting hydroxyls and other polar groups. Subsequent silanization with MEG-TFA residues featuring hydrophobic TFA terminal groups increased hydrophobicity for all coated polymers (CA values ranging from 85 to $91^{\circ}$ ). Finally, the conversion of MEG-TFA into the MEG-OH adlayer with concomitant re-exposure of polar (i.e. hydroxyl) moieties resulted in a substantial increase in surface hydrophilicity, as revealed by a decrease in CA (91$101^{\circ}$, depending on the polymer). These CA measurements match well with the changes in wettability associated with the various modifications of surface functionalities and are entirely consistent with the data obtained via XPS characterization. 


\subsection{Fluorescence Microscopy of Polymers Exposed to Blood}

Using fluorescence microscopy, we analyzed PC, PVC and PS after exposure to blood at a shear rate of $\sim 20 \mathrm{~s}^{-1}$. This shear rate is rather low; however, low shear rates can be found in the human body (e.g. the vena cava, which can have a shear rate as low as $5 \mathrm{~s}^{-1}$ [33-35]) as well as within medical equipment, which we calculated to be $\sim 12 \mathrm{~s}^{-1}$. In our previous studies we focused on high shear rates (e.g. 300$\left.1500 \mathrm{~s}^{-1}\right)$; however, for this investigation we chose a lower shear rate, which is in the applicable range for implants and medical equipment, in order to examine the effect on blood-surface interactions.

Fluorescence spectroscopy analysis of surfaces, bare or MEG-OH-coated, before contact with blood revealed the presence of several background spots that potentially could be erroneously ascribed as originating from blood thrombosis, especially on PC and PVC (Figure 3). In these cases, it is likely that these fluorescence sources originate from either defects in the material or deposited foreign materials, most commonly fungus, could originate from the air during storage or transport and may release fluorescent light when exposed to excitation wavelength extracted by the microscope [36-38]. With respect to defects caused by scratches or dents, they can result in reflected radiation rendering an appearance that can be mistaken for genuine fluorescence. Most scratches had a linear appearance and were avoided during scans. Fluorescent spots are more clearly seen on PVC and PC due to the high transparency of these polymers. On PS, spots were almost non-existent due to the high background signal that overshadows any fluorescence associated with other sources (Figures $3 \mathrm{C}$ and $3 \mathrm{~F}$ ). For this material, background fluorescence is associated with conjugated sulfur-oxygen bonds and aromatic rings. The spots, in most cases, do not significantly contribute to a high signal intensity compared to the fluorescence signal later observed due to blood exposure.
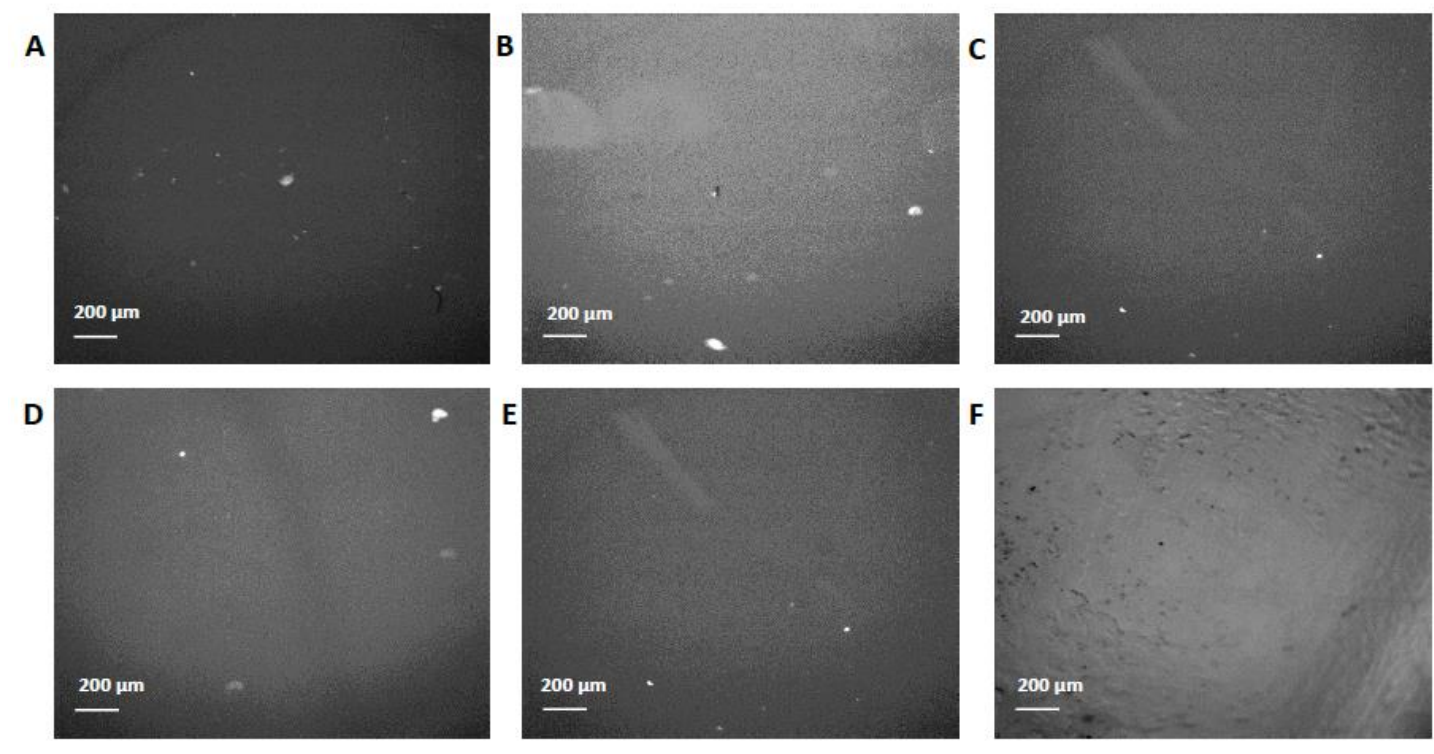

Figure 3 Representative fluorescent images of different surfaces (4× magnification) prior to blood contact. Top (bare surfaces): A) PC, B) PVC, and C) PS; bottom (MEG-OH-coated surfaces): D) PC, E) PVC, and F) PS. Dark areas represent defect- or contamination-free surfaces. 
Following 3 hours of exposure to blood, all MEG-OH derivatized surfaces were observed to have significantly lower platelet surface coverage than their corresponding bare counterparts (Figure 4, Table 2). On PC and PVC, as found in our previous studies $[25,26]$, the reduction was observed to be remarkably high at values of $88 \%$ and $90 \%$, respectively (Table 3 ). When measured with ImageJ, the platelet aggregate diameter appears to be below $200 \mu \mathrm{m}$ and very uncommon on both PC and PVC MEG-OH-coated surfaces (Figures 4D and 4E) compared to much larger aggregates ( $400 \mu \mathrm{m}$ ) on bare PC and PVC (Figures 4A and 4B)). On the other hand, experiments on PS, both bare and MEG-OH-coated, yielded no large clots (Figures $4 \mathrm{C}$ and $4 \mathrm{~F}$ ) with all clots being approximately only a few micrometers in size. In contrast, the reduction in coverage for PS was much lower at $44 \%$.
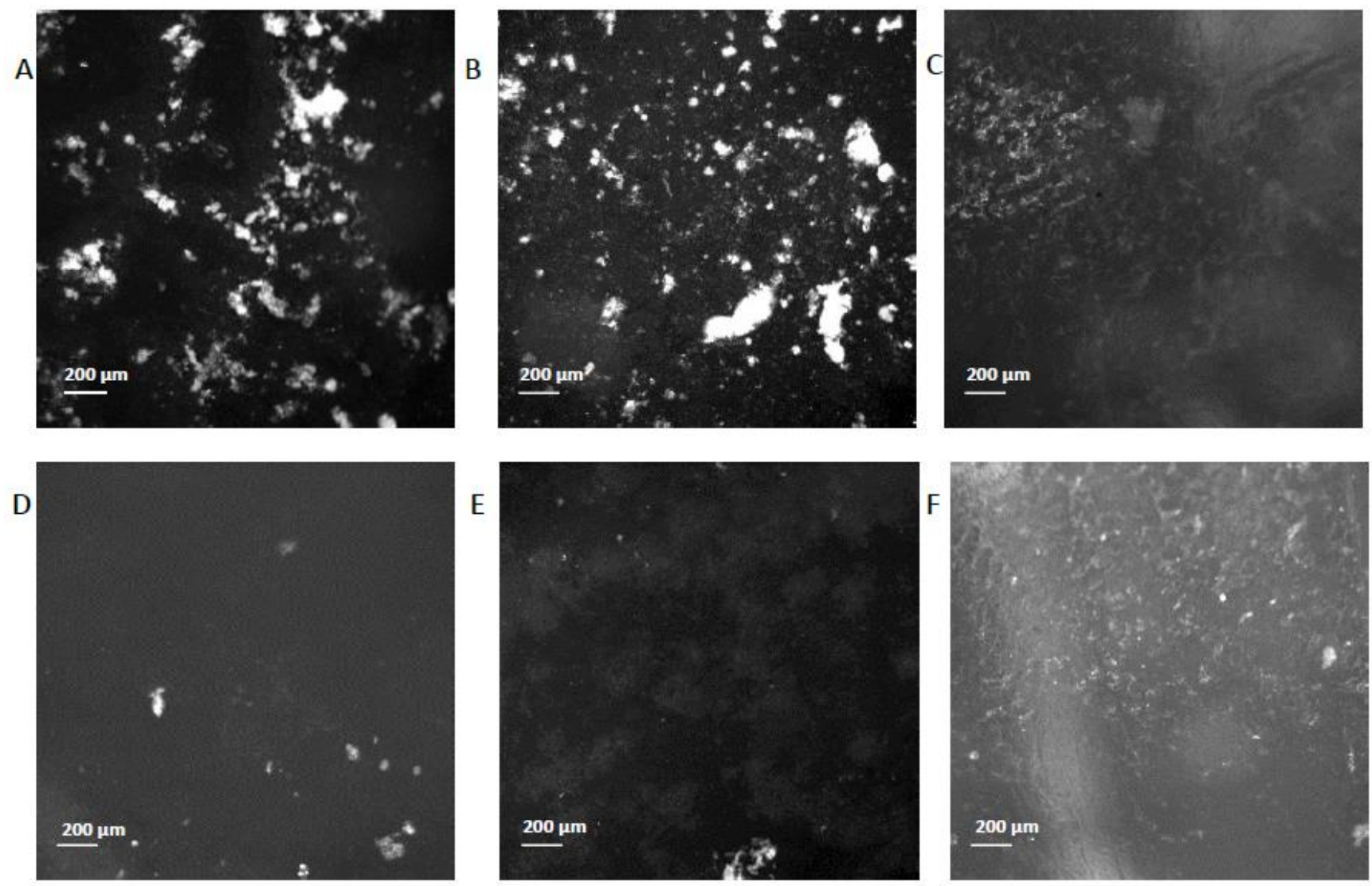

Figure 4 Representative fluorescent images of different surfaces (4× magnification) after 3 hours of blood contact. Top (bare surfaces): A) PC, B) PVC, and C) PS; bottom (MEG-OHcoated surfaces): D) PC, E) PVC, and F) PS. Dark areas represent(platelet-free) polymer surfaces (bare or MEG-OH-derivatized), and the bright areas represent attached fluorescently-labelled platelets. 
Table 2 Compilation of surface coverage percentage due to platelet adhesion, aggregation, and thrombus formation on bare and MEG-OH-coated PC, PVC, and PS following blood contact for 3 hours, 6 hours, and 3 days. A minimum of three replicates were performed for each.

\begin{tabular}{lllll}
\hline & & \multicolumn{2}{l}{ PC surface } & \multicolumn{2}{l}{ PVC surface } & \multicolumn{2}{l}{ PS surface } \\
& & $\begin{array}{l}\text { (\% surface } \\
\text { coverage) }\end{array}$ & $\begin{array}{l}\text { (\% surface } \\
\text { coverage) }\end{array}$ & $\begin{array}{l}\text { (\% surface } \\
\text { coverage) }\end{array}$ \\
\hline \multirow{2}{*}{ No blood exposure } & Bare & $0.4 \pm 0.1$ & $0.6 \pm 0.1$ & $0.1 \pm 0.1$ \\
& MEG-OH adlayer & $0.8 \pm 0.3$ & $1.0 \pm 0.6$ & $0.0 \pm 0.0$ \\
\hline \multirow{2}{*}{ h hours blood exposure } & Bare & $10.0 \pm 1.0$ & $7.8 \pm 0.8$ & $12.0 \pm 4.0$ \\
& MEG-OH adlayer & $1.2 \pm 0.2$ & $0.8 \pm 0.2$ & $7.0 \pm 1.0$ \\
\hline \multirow{2}{*}{ hours blood exposure } & Bare & $2.3 \pm 0.7$ & $3.6 \pm 0.7$ & $2.2 \pm 0.5$ \\
& MEG-OH adlayer & $0.4 \pm 0.1$ & $0.2 \pm 0.0$ & $0.3 \pm 0.2$ \\
\hline \multirow{2}{*}{ days blood exposure } & Bare & $68.0 \pm 4.0$ & $6.0 \pm 1.0$ & $21.0 \pm 3.0$ \\
& MEG-OH adlayer & $6.0 \pm 2.0$ & $0.2 \pm 0.1$ & $10.0 \pm 1.0$ \\
\hline
\end{tabular}

Table 3 Percentage reduction by MEG-OH adlayers of surface coverage by platelet aggregates (compared to bare polymer surfaces) when subjected to blood for specific durations.

\begin{tabular}{llll}
\hline & $\begin{array}{l}\text { PC surface } \\
\text { (MEG-OH/Bare \%) }\end{array}$ & $\begin{array}{l}\text { PVC surface } \\
\text { (MEG-OH/Bare \%) }\end{array}$ & $\begin{array}{l}\text { PS surface } \\
\text { (MEG-OH/Bare \%) }\end{array}$ \\
\hline 3 hours & 88 & 90 & 42 \\
6 hours & 82 & 94 & 86 \\
3 days & 89 & 97 & 53 \\
\hline
\end{tabular}

After 6 hours of blood exposure, the same trends were observed as seen for 3 hours blood contact with the reduction of platelet aggregates on MEG-OH coated compared to bare PC and PVC surfaces (Table 2, Figure 5) still being at $82 \%$ for PC and $94 \%$ for PVC (Table 3). In this case, PS had a reduction of $86 \%$, which is significantly lower compared to 3 hours blood exposure. Additionally, compared to 3 hours blood exposure, the clot sizes and platelet aggregate coverage decreased on all coated polymers. The overall observed reduction in surface coverage is likely caused by the removal of large clots during flow or washing procedures. Such entities, in our experience, are more prone to removal by washing and might have been dislodged under flow conditions. 

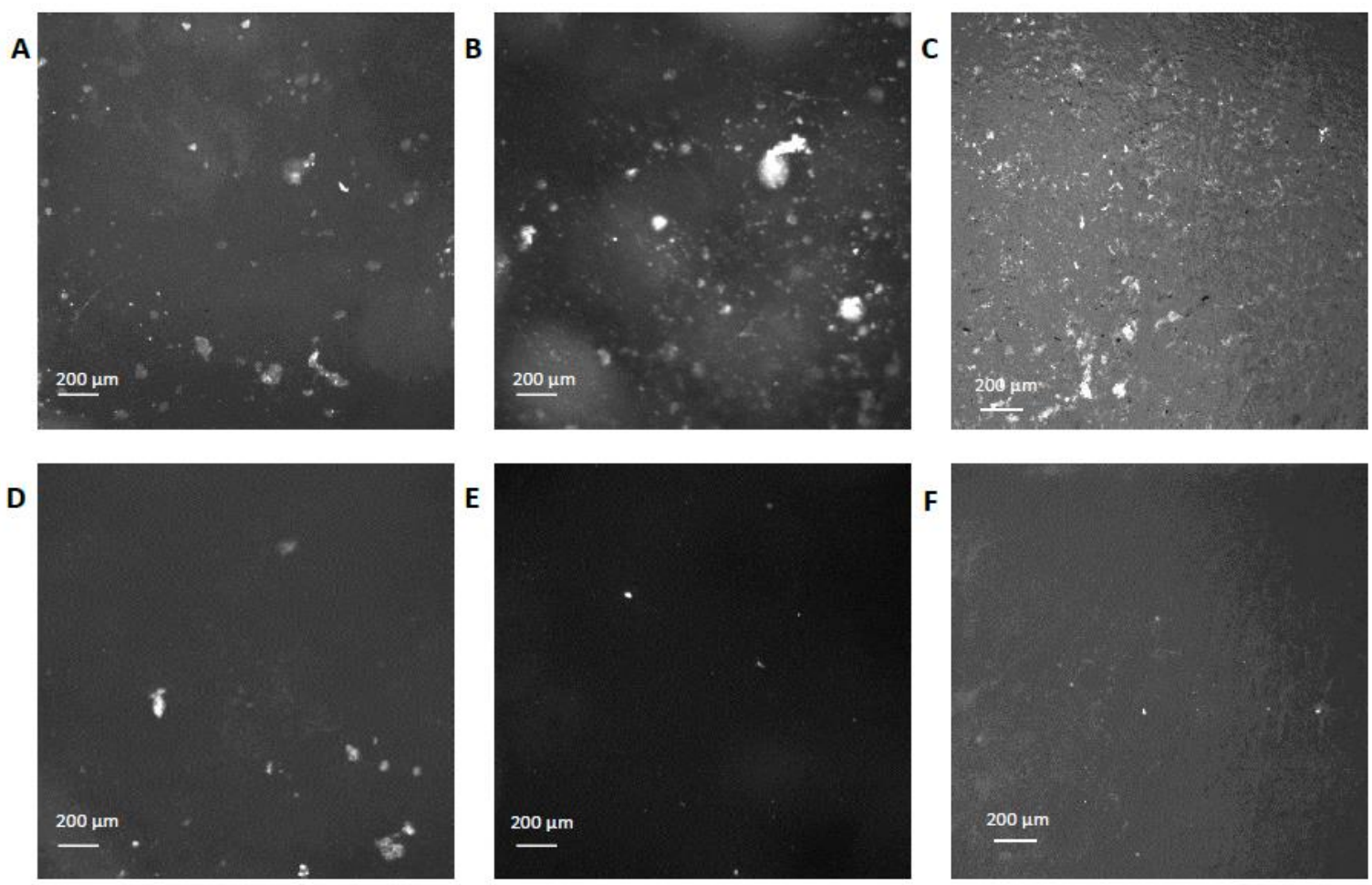

Figure 5 Representative fluorescent images of different surfaces (4× magnification) after 6 hours of blood contact. Top (bare surfaces): A) PC, B) PVC, and C) PS; bottom (MEG-OHcoated surfaces): D) PC, E) PVC, and F) PS. Dark areas represent (platelet-free) polymer surfaces (bare or MEG-OH-derivatized) and the bright areas represent attached fluorescently-labelled platelets.

After 3 days of blood exposure, bare PC and PS substrates exhibited significant platelet aggregation (Figure 6 ) with values of approximately $68 \%$ and $21 \%$, respectively (Table 2 ). Clot sizes were observed to be as large as $1 \mathrm{~mm}$ on PC and $\sim 0.5 \mathrm{~mm}$ on PS; however, for MEG-OH-coated PC and PS, aggregates were much smaller with a size of $\sim 200-300 \mu \mathrm{m}$ (Figure 6). Remarkably, despite the relatively long period of blood contact, the MEG-OH-coated surfaces show reductions of respectively $89 \%$ and $54 \%$ for PC and PS, confirming that the surface modification did not lose efficacy over the specified time period. With respect to PVC, this polymer exhibited similar results to 3 hours blood exposure and a $97 \%$ reduction in platelet aggregates for MEG-OH-coated compared to bare. It should be noted that these results were achieved despite the relatively harsh conditions of our experiments: (1) in a deliberate fashion, no anticoagulants (besides its collection and storage in heparinized Vacutainers) were added to any of the blood aliquots, (2) blood was stored at room temperature with no special cooling to reduce coagulation, and (3) no coating was applied to any of the conduits employed in the flow-through experiments. Even under these conditions, where a level of coagulation could be anticipated, a major reduction in aggregate deposition was observed on all polymers studied. 

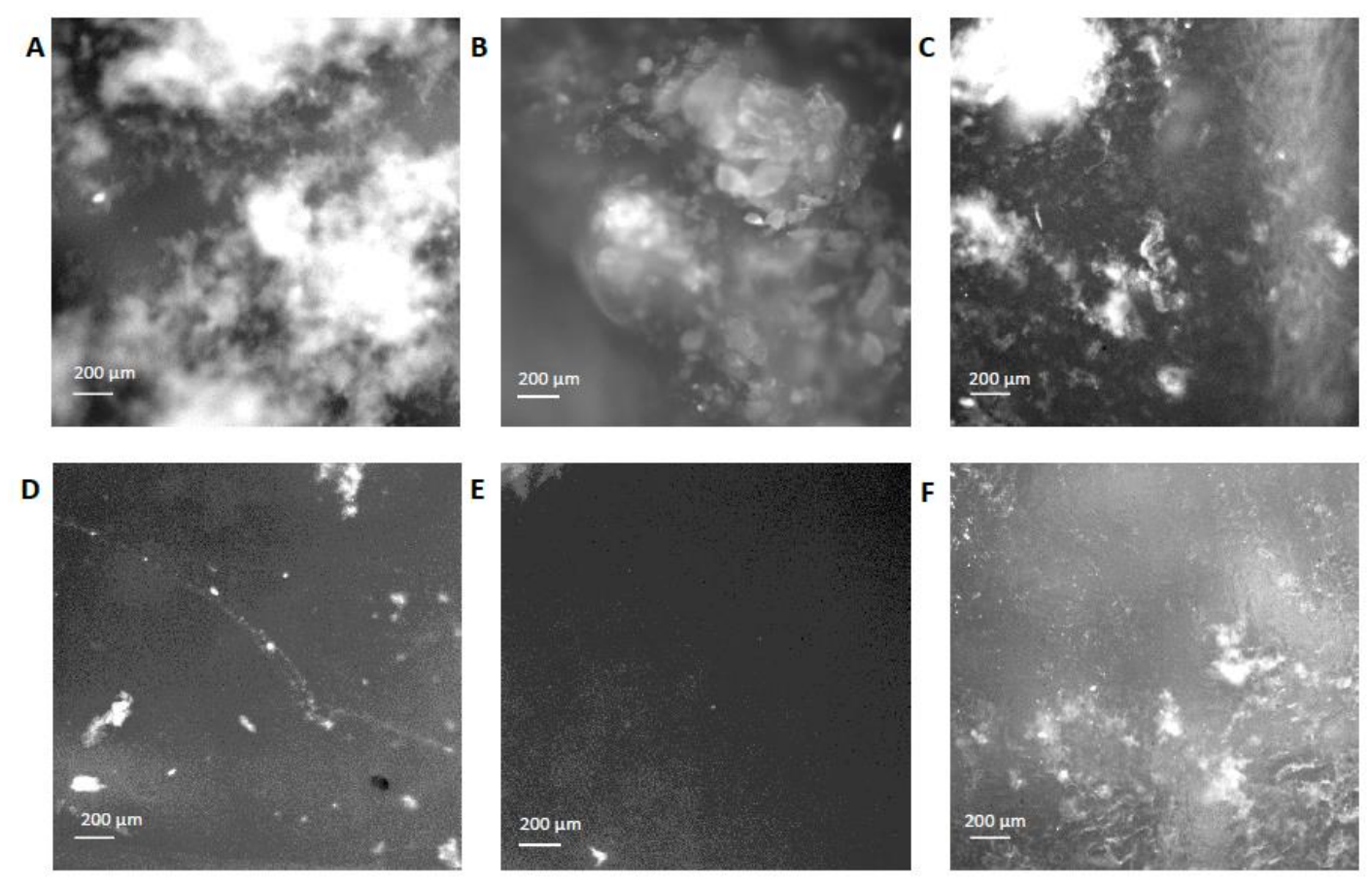

Figure 6 Representative fluorescent images of different surfaces (4× magnification) after 3 days of blood contact. Top (bare surfaces): A) PC, B) PVC, and C) PS; bottom (MEG-OHcoated surfaces): D) PC, E) PVC, and F) PS. Dark areas represent (platelet-free) polymer surfaces (bare or MEG-OH-derivatized) and the bright areas represent attached fluorescently-labelled platelets.

We note that roughness is a possible factor in terms of the instigation of interfacial coagulation for both bare and MEG-OH-coated substrates [26]. PC and PVC have an arithmetical mean roughness (Ra) value of $0.1 \mu \mathrm{m}$, which is standard for manufacturing these polymers. With regards to PS, microscope analysis revealed trough sizes of $\sim 20 \mu \mathrm{m}$ and a Ra estimate of $\sim 3 \mu \mathrm{m}[39,40]$. Although the roughness may not have been a factor on PC or PVC, we see that it may have been an issue for PS since platelet aggregate coverage was less predictable (Table 2). Fouling on polymers is known to be increased by an elevated level of surface roughness $[41,42]$. Thus, the results would be comparable with PVC and PC if PS had a Ra of 0.1 . Moreover, we believe that any coagulation that occurs on MEG-OH-coated polymers was likely the result of the presence of surface defects or areas not coated with MEG-OH. Overall, the best MEG-OH-derivatized polymer system appears to be PVC, PC being a close second. Reduction of platelet surface coverage is observed with PS as well, to a much lesser extent, however.

\section{Final Remarks}

Surface modification of polycarbonate, poly (vinyl chloride), and polysulfone polymeric materials by MEG-OH silane surface chemistry has been shown to be highly effective with regard to the enhancement of hemocompatibility, even for conditions where surfaces were exposed to blood for extended periods of time (up to several days). MEG-OH adlayer on PC and PVC displayed reductions 
ranging between $88-97 \%$ in surface coverage by platelets when compared to bare substrates. PS was noticeably less effective (42-86\%).

The durations of polymer surface exposure to blood employed in this study are quite reflective of those used in various medical procedures as described earlier. For example, PVC conduits are often used for blood flow over 3-4 hours during patient renal dialysis. An added feature of this work is the potential advantage that aggregates of particularly small dimension, which are evident on coated surfaces, are unlikely to cause complications when released into the bloodstream. In this respect, "micro-clots" are considered to be dissolved by the regulatory fibrinolytic system in the body [43]. Finally, it is useful to discuss the potential of this surface modification strategy in terms of possible commercial exploitation. As specified in this research, a high degree of prevention of platelet aggregation has been achieved over quite long periods of time and, furthermore, the physical chemistry that lies behind this result is reasonably well understood. On the other hand, it is difficult to contemplate the large-scale application of silanization chemistry. The key issue then will be whether alternative chemical or physical protocols can be developed which mimic the result of silanization technology.

\section{Acknowledgments}

The authors are appreciative of funding provided by the Natural Sciences and Engineering Research Council of Canada (NSERC) in order to conduct the research described in this paper. The authors also extend their gratitude to Dr. Christophe Blaszykowski for designing and synthesizing the MEG-TFA surface modifier and for fruitful discussions. Special acknowledgements are directed to Dr. Peter M. Brodersen of Surface Interface Ontario for acquisition of the XPS data. Use of the fluorescence microscope at the Krembil Research Institute (Western Hospital of Toronto, Canada) under the auspices of Professor Peter Carlen (University Health Network and Department of Physiology University of Toronto, Canada) is very gratefully acknowledged.

\section{Author Contributions}

M.T. and A.R. conceived the experimental strategy; S.S. devised the MEG-OH surface chemistry; K.F. prepared the various surfaces; K.F. and S.S. analyzed XPS and contact angle data; K.F. performed all blood-polymer interaction and fluorescence microscopy work; M.T. wrote the paper in consultation with K.F., which all co-authors edited.

\section{Conflicts of Interest}

The authors declare no conflict of interest.

\section{References}

1. Surgical Procedure Volumes: Global Analysis with Forecasts to 2030 (US, China, Japan, Brazil, UK, Germany, Italy, France, Mexico, Russia) [Internet]. Arlington: Kalorama; 2014. Available from: 
https://kaloramainformation.com/product/surgical-procedure-volumes-global-analysis-withforecasts-to-2030-us-china-japan-brazil-uk-germany-italy-france-mexico-russia/.

2. Stevens KN, Aldenhoff YB, Van der Veen FH, Maessen JG, Koole LH. Bioengineering of improved biomaterials coatings for extracorporeal circulation requires extended observation of blood biomaterial interaction under flow. J Biomed Biotechnol. 2007; 2007: 29464.

3. Murphy GJ, Angelini GD. Side effects of cardiopulmonary bypass: What is the reality? J Card Surg. 2004; 19: 481-488.

4. Ekdahl KN, Lambris JD, Elwing H, Ricklin D, Nilsson PH, Teramura Y, et al. Innate immunity activation on biomaterial surfaces: A mechanistic model and coping strategies. Adv Drug Deliv Rev. 2011; 63: 1042-1050.

5. Anderson JM. Biological responses to materials. Annu Rev Mater Res. 2001; 31: 81-110.

6. Murray AM. Cognitive impairment in the aging dialysis and chronic kidney disease populations: An occult burden. Adv Chronic Kidney Dis. 2008; 15: 123-132.

7. Stroobant N, Van Nooten G, Van Belleghem Y, Vingerhoets G. Relation between neurocognitive impairment, embolic load, and cerebrovascular reactivity following on-and off-pump coronary artery bypass grafting. Chest. 2005; 127: 1967-1976.

8. Di lorio B, Lopez T, Procida M, Marino P, Valente V, lannuzziello F, et al. Successful use of central venous catheter as permanent hemodialysis access: 84-month follow-up in Lucania. Blood Purif. 2001; 19: 39-43.

9. Flythe JE, Curhan GC, Brunelli SM. Shorter length dialysis sessions are associated with increased mortality, independent of body weight. Kidney Int. 2013; 83: 104-113.

10. Tentori F, Zhang J, Li Y, Karaboyas A, Kerr P, Saran R, et al. Longer dialysis session length is associated with better intermediate outcomes and survival among patients on in-center three times per week hemodialysis: Results from the Dialysis Outcomes and Practice Patterns Study (DOPPS). Nephrol Dial Transplant. 2012; 27: 4180-4188.

11. Bellomo R, Ronco C. Continuous renal replacement therapy in the intensive care unit. Intensive Care Med. 1999; 25: 781-789.

12. Salis S, Mazzanti VV, Merli G, Salvi L, Tedesco CC, Veglia F, et al. Cardiopulmonary bypass duration is an independent predictor of morbidity and mortality after cardiac surgery. J Cardiothorac Vasc Anesth. 2008; 22: 814-822.

13. Napalkov P, Felici DM, Chu LK, Jacobs JR, Begelman SM. Incidence of catheter-related complications in patients with central venous or hemodialysis catheters: A health care claims database analysis. BMC Cardiovasc Disord. 2013; 13: 86.

14. Pinon M, Bezzio S, Tovo PA, Fagioli F, Farinasso L, Calabrese R, et al. A prospective 7-year survey on central venous catheter-related complications at a single pediatric hospital. Eur J Pediatr. 2009; 168: 1505-1512.

15. Lee AY, Levine MN, Butler G, Webb C, Costantini L, Gu C, et al. Incidence, risk factors, and outcomes of catheter-related thrombosis in adult patients with cancer. J Clin Oncol. 2006; 24: 1404-1408.

16. Cortelezzi A, Moia M, Falanga A, Pogliani EM, Agnelli G, Bonizzoni E, et al. Incidence of thrombotic complications in patients with haematological malignancies with central venous catheters: $A$ prospective multicenter study. Br J Haematol. 2005; 129: 811-817. 
17. Sastri VR. Plastics in medical devices: Properties, requirements, and applications. 2nd ed. Norwich: William Andrew; 2013.

18. Powell DG. Medical applications of polycarbonate douglas. Med Plast Biomate Mag. 1998; 5: 3845.

19. Sastri VR. Engineering thermoplastics: Acrylics, polycarbonates, polyurethanes, polyacetals, polyesters, and polyamides. Plastics in medical devices: Properties, requirements and applications. 2nd ed. Norwich: William Andrew; 2013.

20. Thompson M, Blaszykowski C, Sheikh S, Rodriguez-Emmenegger C, De los Santos Pereira A. Biological-fluid surface interactions in detection and medical devices. Cambridge: Royal Society of Chemistry Publishing; 2016.

21. De los Santos Pereira A, Riedel T, Brynda E, Rodriguez-Emmenegger C. Hierarchical antifouling brushes for biosensing applications. Sens Actuators B Chem. 2014; 202: 1313-1321.

22. De Mel A, Cousins BG, Seifalian AM. Surface modification of biomaterials: A quest for blood compatibility. Int J Biomater. 2012; 2012: 707863.

23. Blaszykowski C, Sheikh S, Thompson M. A survey of state-of-the-art chemistries to minimize fouling from human and animal biofluids. Biomater Sci. 2015; 3: 1335-1370.

24. Sheikh S, Yang DY, Blaszykowski C, Thompson M. Single ether group in glycol- based ultra-thin layer monolayer prevents surface fouling from undiluted serum. Chem Commun. 2012; 48: 1305-1307.

25. Fedorov K, Blaszykowski C, Sheikh S, Reheman A, Romaschin A, Ni H, et al. Prevention of thrombogenesis from whole human blood on plastic polymer by ultrathin monoethylene glycol silane adlayer. Langmuir. 2014; 30: 3217-3222.

26. Fedorov K, Jankowski A, Sheikh S, Blaszykowski C, Reheman A, Romaschin A, et al. Prevention of surface-induced thrombogenesis on polyvinyl chloride. J Mater Chem B. 2015; 3: 8623-8628.

27. Pawlowska NM, Fritzsche H, Blaszykowski C, Sheikh S, Vezvaie M, Thompson M. Probing the hydration of ultra-thin antifouling silane adlayers using neutron reflectometry. Langmuir. 2014; 30 : 1199-1203.

28. Sheikh S, Blaszykowski C, Nolan R, Thompson D, Thompson M. On the hydration of subnanometric antifouling organosilane adlayers: A molecular dynamics simulation. J Colloid Interface Sci. 2015; 437: 197-204.

29. Sheikh S, Sheng JC, Blaszykowski C, Thompson M. New oligoethylene glycol linkers for the surface modification of an ultra-high frequency acoustic wave biosensor. Chem Sci. 2010; 1: 271-275.

30. Decker C, Balandier M. Photo-oxidation of poly (vinyl chloride). Polym Photochem. 1981; 1: 221232.

31. Artham T, Doble M. Biodegradation of aliphatic and aromatic polycarbonates. Macromol Biosci. 2008; 8: 14-24.

32. Rivaton A, Gardette JL. Photodegradation of polyethersulfone and polysulfone. Polym Degrad Stabil. 1999; 66: 385-403.

33. Lipowsky HH, Kovalcheck ST, Zweifach BW. The distribution of blood rheological parameters in the microcirculation of cat mesentery. Circ Res. 1978; 43: 738-749.

34. Fahraeus R, Lindqvist T. The viscosity of blood in narrow capillary tubes. Am J Physiol. 1931; 96 : 562-568. 
35. Papaioannou TG, Stefanadis C. Vascular wall shear stress: Basic principles and methods. Hellenic J Cardiol. 2005; 46: 9-15.

36. Fröhlich-Nowoisky J, Pickersgill DA, Després VR, Pöschl U. High diversity of fungi in air particulate matter. Proc Natl Acad Sci U S A. 2009; 106: 12814-12819.

37. Bauer H, Schueller E, Weinke G, Berger A, Hitzenberger R, Marr IL, et al. Significant contributions of fungal spores to the organic carbon and to the aerosol mass balance of the urban atmospheric aerosol. Atmos Environ. 2008; 42: 5542-5549.

38. Elbert W, Taylor PE, Andreae MO, Pöschl U. Contribution of fungi to primary biogenic aerosols in the atmosphere: Wet and dry discharged spores, carbohydrates, and inorganic ions. Atmos Chem Phys. 2007; 7: 4569-4588.

39. Surface roughness. Huangyang: Plastic mold company and China plastic mold company; 2020. Available from: http://www.myplasticmold.com/surface-roughness.html

40. Ramsdale R. Engineers online handbook. Table of surface roughness. Available from: http://www.engineershandbook.com/Tables/surfaceroughness.htm

41. Yamamoto T, Umegae S, Matsumoto K. Safety and clinical efficacy of granulocyte and monocyte adsorptive apheresis therapy for ulcerative colitis. World J Gastroenterol. 2006; 12: 520-525.

42. Jimenez M, Hamze H, Allion A, Ronse G, Delaplace G, Traisnel M. Antifouling stainless steel surface: Competition between roughness and surface energy. Mater Sci Forum. 2012; 706-709: 2523-2528.

43. Hudson NE. Biophysical mechanisms mediating fibrin fiber lysis. BioMed Res Int. 2017; 2017: 2748340.

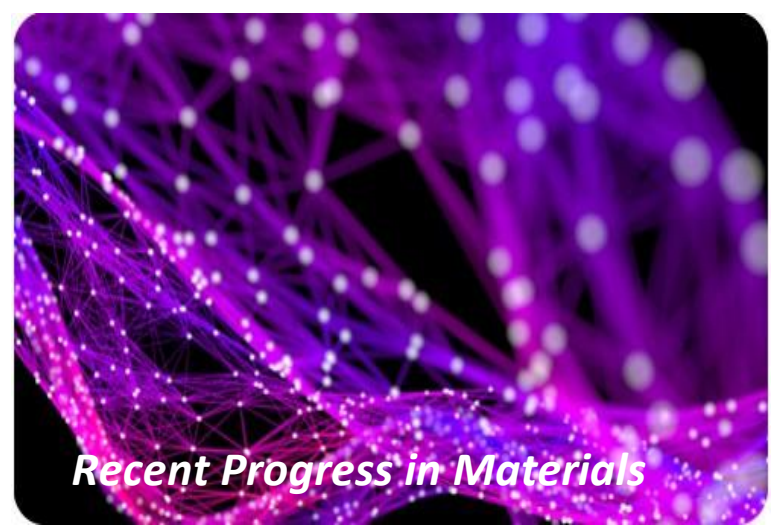

Enjoy Recent Progress in Materials by:

1. Submitting a manuscript

2. Joining in volunteer reviewer bank

3. Joining Editorial Board

4. Guest editing a special issue

For more details, please visit:

http://www.lidsen.com/journals/rpm 